\title{
Are pollination syndromes useful predictors of floral visitors in Tasmania?
}

\author{
ANDREW B. HINGSTON` AND PETER B. MC QUILLAN \\ Geography and Environmental Studies, University of Tasmania, GPO Box 252-78, Hobart, Tasmania \\ 7001, Australia (Email: Andrew.Hingston@utas.edu.au)
}

\begin{abstract}
Diurnal visitors to the flowers of many native plant species were identified in a wide range of Tasmanian sclerophyllous vegetation between September 1996 and April 1997. These foraging profiles were analysed to determine whether they were characteristic of various floral morphologies in predictable ways. It was found that although visitor profiles were sometimes consistent with classic pollination syndromes, these syndromes were unreliable predictors of floral visitors. Very few flowers were exclusively bird-pollinated, and none were strictly fly-, beetle-, wasp-, or butterfly-pollinated. The majority of flowering plants were unspecialized in their morphology, and consequently hosted a diverse array of visitors. In addition, visitor profiles to congeners with similar floral morphologies, and even to conspecifics, differed between habitats. Altitude was a major factor in determining visitors, with flies being the most abundant visitors above $700 \mathrm{~m}$. However, congeners in several genera of Epacridaceae, as well as the genus Correa, which differed in floral morphology also differed in visitor profiles. Tubular flowers were associated with birds, while flowers with more accessible nectar were visited by insects. The only taxa exhibiting a bee-pollination syndrome that were largely visited by bees were the Fabaceae and Goodenia ovata Sm. Several species with purple or pink flowers were also predominantly visited by bees, but did not strictly conform to the melittophilous syndrome. In contrast, other flowers exhibiting an ostensibly mellitophilous syndrome hosted very few bees. Of these, species that occurred at high altitude were mainly visited by flies, while others received very few potential pollen vectors.
\end{abstract}

Key words: birds, colour, flowers, foraging profiles, insects, shape, visitor profiles.

\section{INTRODUCTION}

It has been proposed that zoophilous flowers exhibit different syndromes that relate to their particular animal pollinators (Faegri \& van der Pijl 1979). Such flowers exhibit varying degrees of specialization to their pollen vectors, ranging from allophilic flowers, which may be pollinated by any visitor, to euphilic flowers, which restrict access to floral rewards to highly specialized coevolved pollinators (Faegri \& van der Pijl 1979).

Beetles, flies and wasps are generally restricted to foraging on allophilic flowers, which are typically dull coloured, actinomorphic, and have exposed floral rewards. The beetle-pollinated (cantharophilous) syndrome also includes a strong odour but not nectar guides, in contrast to the fly-pollinated (myophilous) syndrome which includes nectar guides but no scent (Faegri \& van der Pij1 1979). However, Faegri and van der Pijl (1979) did not regard the definition of a wasppollination syndrome (sphecophily) as a possibility because of the lack of uniformity in characteristics of flowers visited by this group.

${ }^{\star}$ Corresponding author.

Accepted for publication February 2000.
While butterflies, birds and bees can also forage from allophilic flowers, they have exclusive access to euphilic flowers exhibiting their particular syndromes. Possession of a long proboscis in butterflies allows them to access nectar from narrow tubular corollas, in addition to those with more exposed nectar. These butterfly-pollinated (psychophilous) flowers are typically brightly coloured with little scent (Faegri \& van der Pijl 1979). Bird-pollinated (ornithophilous) flowers are varied in form, and include the brush, gullet and tubular types, as well as some flag-blossoms of the Fabaceae. They produce large volumes of nectar and are often bright red (Faegri \& van der Pijl 1979). In tubular flowers, the floral tube is wider in bird than in butterfly-pollinated flowers (Cruden \& HermannParker 1979; Faegri \& van der Pijl 1979). It has also been proposed that butterfly-pollinated flowers have a yellow target indicating the whereabouts of nectar against a red background, whereas bird-pollinated flowers tend to have yellow margins or tips (Cruden \& Hermann-Parker 1979). Flowers typical of pollination by the bee family Apidae (melittophilous) are zygomorphic, bright yellow or blue with nectar guides, and frequently with hidden rewards such as those in the Lamiaceae, Scrophulariaceae, Fabaceae and Orchidaceae (Faegri \& van der Pijl 1979). 
The concept of pollination syndromes has been adopted by numerous authors. Syndromes not investigated in this paper include pollination by non-flying mammals (Rourke \& Wiens 1977), bat-pollination (chiropterophily) (Faegri \& van der Pijl 1979), antpollination (myrmecophily) (Armstrong 1979; Wyatt 1981), thrip-pollination (thripsophily) (Kirk 1988), and microcantharophily (small-beetle-pollination) (Armstrong \& Irvine 1989).

However, Faegri and van der Pijl (1979) stressed that pollination syndromes were only generalizations and that anthophiles, other than those associated with the pollination syndrome of a plant, may also visit its flowers. Further doubts into the predictive value of these floral syndromes have recently been raised. Their universality has been questioned because many species are pollinated by more than one insect order (C. Herrera 1996), insect visitors to conspecific flowers vary at the level of order between microclimates (Rozzi et al. 1997), coblooming plants with different floral morphologies tend to share the same visitors (J. Herrera 1988), and the corolla colour preferences exhibited by insect taxa differ between habitats (McCall \& Primack 1992).

This study aimed to test the applicability of the syndrome concept to flowering plants in a range of vegetation types in Tasmania. As syndromes are based on the secondary floral attractants of colour, shape and smell, it is implied that the different pollinators of plants in each syndrome result from the propensity for animals to visit certain types of flowers. Hence, our investigation of pollination syndromes is based on visitor profiles.

\section{METHODS}

\section{Data collection}

Associations between plants and diurnal flower visitors were investigated in a wide variety of vegetation types in Tasmania (Table 1) between September 1996 and April 1997. We recorded the identities of all visitors, excluding ants, seen on each plant species while walking along tracks. Surveys were conducted between 09.00 hours and 18.00 hours on 83 mild, warm or hot, sunny days with little wind.

Insect specimens were captured for identification by netting them as they foraged at flowers, and depositing them individually in labelled plastic bags. At the end of the day, insects were killed by placing a few drops of ethyl acetate on a piece of rag in the bag and then leaving them for 2 hours. The specimens were subsequently pinned and identified to family under a dissecting microscope using the keys in Borror et al. (1981). Bees were identified to subgenus using the key of Michener (1965), and to species by comparing specimens with the large reference collection at the Museum of Victoria, and using the key of Walker (1995) for Lasioglossum (Chilalictus) spp. Any insects which could not be identified to species level were grouped according to morphospecies. Voucher specimens have been retained at the School of Geography \& Environmental Studies at the University of Tasmania in Hobart, with some Halictidae also deposited at the Museum of Victoria. All plants were identified according to Curtis (1963, 1967) and Curtis and Morris (1975, 1994), but the more recent species nomenclature of Buchanan (1995) was used.

\section{Data analysis}

All visitors were categorized into the taxonomic groups associated with the syndromes espoused by Faegri and van der Pijl (1979). As these authors did not include Hemiptera in their floral syndromes, and this order very rarely visited flowers, these insects were excluded from our analysis. Relationships between plant species on the basis of visitor profiles were explored with an ordination method in which similarity based on visitor profiles is a function of distance apart in the resulting plot. The ordination method chosen was semistrong hybrid multidimensional scaling (SSH MDS), within the computer program PATN (Belbin 1993). The Bray-Curtis index of similarity, based on untransformed proportions of species within a visitor category out of all visitors for that plant species, was used as input to the ordination. The correlation of these visitor categories with the ordination of the plant species was determined (PCC module in PATN; Pattern Analysis Package, CSIRO, Canberra) and the significant groups, as determined by a Monte Carlo technique, were fitted to the ordination plot as vectors. Plants receiving fewer than five visitors were excluded from the analysis because their recorded visitor profiles were often artificially narrow as a consequence of insufficient observations. As this may have excluded some specialized flowers from the analysis, species that were consistently visited by a narrow range of insects have been considered in the discussion.

\section{RESULTS}

\section{Flower visitors observed}

Forty families of insects from five orders, along with three families of birds, were observed visiting flowers in this study. These encompass many of the visitor groups associated with the floral syndromes documented by Faegri and van der Pijl (1979).

All of the groups were significant predictors of the variation among plants in their visitor profiles (Fig. 1). 
The various insect groups were associated with divergent vectors in the plane described by the two axes associated with the greatest variation in visitor profiles.
The vector describing the foraging profile of birds was very similar to that of butterflies, but opposite to that of wasps. Bees and flies also described opposing
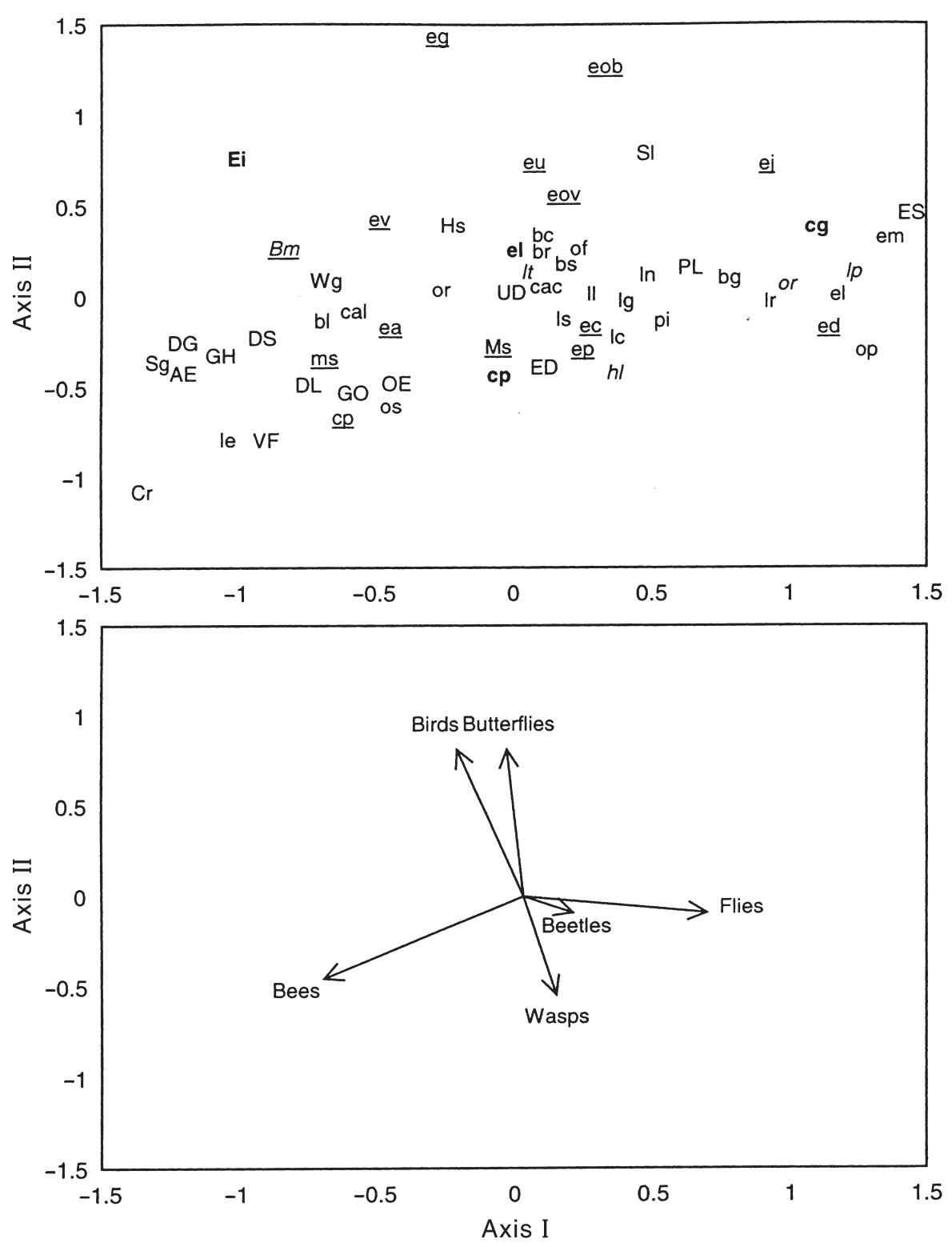

Fig. 1. Ordination of plant species according to their flower visitor profiles. Only those plants visited by at least four species have been included in the analysis. The anthophile groups which were significant $(P<0.05)$ predictors of the variation in flower visitor profiles have been fitted as vectors, shown in a separate plot for clarity. Stress for three axes = $9.65 \%$. Binomial codes for floral morphology: first letter capital $=$ brightly coloured or nectar guides on a pale background; first letter lower case $=$ pale coloured; second letter capital $=$ zygomorphic architecture; second letter lower case $=$ actinomorphic architecture including the capitula of Asteraceae; bold $=$ tubular, where the corolla tube is longer than the corolla lobes; underline $=$ brush, where the anthers form the bulk of the visual display; and italic $=$ Proteaceae. Binomial codes for plant species: $\mathrm{AE}=$ Aotus ericoides (Vent.) G. Don; $\mathrm{bc}=$ Boronia citriodora Gunn ex Hook. f.; bg = Baeckea gunniana Schauer ex Walp.; $\mathrm{bl}=$ Baeckea leptocaulis Hook. f.; $\underline{B m}=$ Banksia marginata Cav.; br = Bauera rubioides Andr.; bs =Bursaria spinosa Cav.; cac $=$ Cassinia aculeata R. Br.; cal = Correa alba Andr.; cg = Cyathodes glauca Labill.; $\mathbf{c p}=$ Cyathodes parvifolia $\mathrm{R}$.

Br.; $\underline{\mathrm{c}}=$ Callistemon pallidus (Bonpl.) DC.; $\mathrm{Cr}=$ Carpobrotus rossii Schwartes; $\mathrm{DG}=$ Dillwynia glaberrima $\mathrm{Sm} . ; \mathrm{DL}=$ Daviesia latifolia R. Br.; DS = Dillwynia sericea A. Cunn.; ea = Eucalyptus (Monocalyptus) amygdalina Labill.; ec = Eucalyptus (Monocalyptus) coccifera Hook. f.; ed = Eucalyptus (Monocalyptus) delegatensis R. T. Baker; ED=Euphrasia collina ssp. diemenica Spreng.; eg = Eucalyptus (Symphyomyrtus) globulus Labill.; Ei = Epacris impressa Labill. (some populations also have white flowers); $\underline{\mathrm{ej}}=$ Eucalyptus (Symphyomyrtus) johnstonii Maiden; el = Epacris lanuginosa Labill.; el = Eucryphia lucida (Labill.) Baill.; em = Eucryphia milliganii Hook. f.; eob = Eucalyptus (Monocalyptus) obliqua L'Herit.; eov = Eucalyptus (Symphyomyrtus) ovata Labill.; $\underline{\mathrm{ep}}=$ Eucalyptus (Monocalyptus) pulchella Desf.; ES = Euphrasia striata R. Br.; eu = Eucalyptus (Symphyomyrtus) urnigera

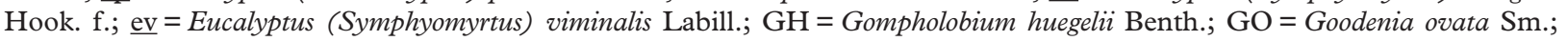
$h l=$ Hakea lissosperma R. Br.; Hs = Helichrysum scorpioides Labill.; lc = Leucopogon collinus $($ Labill.) R. Br.; le = Leucopogon ericoides $(\mathrm{Sm}$.) R. Br.; $1 \mathrm{~g}=$ Leptospermum glaucescens S. Schauer; $11=$ Leptospermum lanigerum (Ait.) Sm.; $\ln =$ Leptospermum nitidum Hook. f.; lp = Lomatia polymorpha R. Br.; lr= Leptospermum rupestre Hook. f.; ls = Leptospermum scoparium J. R. \& G. Forst.; $l t=$ Lomatia tinctoria $\mathrm{R}$. Br.; $\underline{\mathrm{Ms}}=$ Melaleuca squamea Labill.; $\underline{\mathrm{ms}}=$ Melaleuca squarrosa Donn ex Sm.; OE = Oxylobium ellipticum (Labill.) R. Br.; of = Ozothamnus ferrugineus DC.; op = Olearia phlogopappa (Labill.) DC.; or = Olearia ramulosa (Labill.) Benth.; or $=$ Orites revoluta $\mathrm{R}$. Br.; os = Olearia stellulata (Labill.) DC.; pi = Pentachondra involucrata R. Br.; PL = Prostanthera lasianthos Labill.; Sg = Stylidium graminifolium Swartz; Sl = Senecio linearifolius A. Rich.; UD = Utricularia dichotoma Labill.; VF = Veronica formosa R. Br.; Wg = Wahlenbergia gymnoclada N. Lothian. 
Table 1. Summary of study sites

\begin{tabular}{|c|c|c|c|}
\hline Vegetation type & Coordinates & Altitude (m) & Rainfall (mm) \\
\hline \multirow[t]{2}{*}{ Coastal heathy woodland } & $43.03^{\circ} \mathrm{S}, 147.50^{\circ} \mathrm{E}$ & 10 & $550-750$ \\
\hline & $43.02^{\circ} \mathrm{S}, 147.30^{\circ} \mathrm{E}$ & $10-100$ & $550-750$ \\
\hline Grassy woodland & $42.81^{\circ} \mathrm{S}, 147.26^{\circ} \mathrm{E}$ & 5 & 550 \\
\hline \multirow[t]{7}{*}{ Dry sclerophyll forest } & $43.04^{\circ} \mathrm{S}, 147.18^{\circ} \mathrm{E}$ & 140 & $750-1000$ \\
\hline & $42.93^{\circ} \mathrm{S}, 147.30^{\circ} \mathrm{E}$ & $200-250$ & 750 \\
\hline & $42.92^{\circ} \mathrm{S}, 147.30^{\circ} \mathrm{E}$ & $150-350$ & 1000 \\
\hline & $42.91^{\circ} \mathrm{S}, 147.29^{\circ} \mathrm{E}$ & $230-240$ & 1000 \\
\hline & $42.67^{\circ} \mathrm{S}, 146.75^{\circ} \mathrm{E}$ & 300 & $750-1000$ \\
\hline & $41.62^{\circ} \mathrm{S}, 146.61^{\circ} \mathrm{E}$ & $450-500$ & $1250-1500$ \\
\hline & $41.54^{\circ} \mathrm{S}, 146.47^{\circ} \mathrm{E}$ & $200-300$ & $1000-1250$ \\
\hline \multirow[t]{3}{*}{ Lowland wet scrub } & $43.10^{\circ} \mathrm{S}, 146.72^{\circ} \mathrm{E}$ & 60 & $1250-1500$ \\
\hline & $42.90^{\circ} \mathrm{S}, 146.37^{\circ} \mathrm{E}$ & 320 & 2000 \\
\hline & $42.84^{\circ} \mathrm{S}, 146.55^{\circ} \mathrm{E}$ & 260 & 1250 \\
\hline \multirow[t]{3}{*}{ Subalpine mixed forest } & $43.20^{\circ} \mathrm{S}, 146.84^{\circ} \mathrm{E}$ & $520-800$ & $1500-2500$ \\
\hline & $42.90^{\circ} \mathrm{S}, 147.24^{\circ} \mathrm{E}$ & $720-1000$ & $1250-1500$ \\
\hline & $42.67^{\circ} \mathrm{S}, 146.61^{\circ} \mathrm{E}$ & $920-1060$ & 1500 \\
\hline
\end{tabular}

vectors, while the beetle vector was associated with the centre of the plot (Fig. 1).

\section{Plant visitor profiles}

The plant species were scattered across the plot, with no distinct clusters and no clear divisions between different floral morphologies (Fig. 1). Brush-like inflorescences and tubular flowers were both scattered very widely across the plot, indicating that neither of these characteristics were associated with particular categories of floral visitors. However, a high proportion of the species situated on the left of the plot, and hence associated with bees, exhibited brightly coloured flowers. These included all of the Fabaceae as well as some other flowers with zygomorphic architecture. Nevertheless, there were some brightly coloured and some zygomorphic flowers in the right half of the plot. Furthermore, the pale yellow flowers of Melaleuca squarrosa Donn ex Sm. were more strongly associated with bees than were the purple flowers of Melaleuca squamea Labill. (Fig. 1).

All of the plants with values greater than 0.5 on Axis I, except Eucryphia lucida, occurred at high altitude $(700-1100 \mathrm{~m})$. Several of these species associated with flies were positioned further to the right than their low-altitude $(<350 \mathrm{~m})$ congeners with similar floral morphologies. This pattern was apparent in the high altitude species Lomatia polymorpha R. Br., Olearia phlogopappa (Labill.) DC., Baeckea gunniana Schauer ex Walp., Eucalyptus johnstonii Maiden and Leptospermum rupestre Hook. f. (Fig. 1 \& Table 2). Although both Euphrasia striata R. Br. and Euphrasia collina ssp. diemenica (Sprengel.) W. R. Barker occurred on Mt Wellington (720-1100 m), the former was positioned further to the right on the plot and occu- pied higher altitude areas within this range in accordance with this trend. However, this altitudinal difference in visitor profiles between congeners with similar floral morphologies was not apparent in Eucryphia (Fig. 1 \& Table 2).

Visitor profiles usually differed between congeners occurring at similar altitudes. These included members of the Epacridaceae genera Cyathodes, Leucopogon and Epacris. Cyathodes glauca Labill. exhibited strong affinities with flies, Leucopogon ericoides (Sm.) R. Br. and Leucopogon virgatus (Labill.) $\mathrm{R}$. Br. with bees, and Epacris impressa Labill. with bees and birds, whereas Cyathodes parvifolia R. Br., L. collinus (Labill.) R. Br. and Epacris lanuginosa Labill. were less specialized. However, Melaleuca and Dillwynia congeners did not differ markedly in visitor profiles (Fig. 1 \& Table 2). Differing visitor profiles between congeners were also apparent in Eucalyptus. All species in the subgenus Symphyomyrtus were placed in the top half of the ordination space, whereas all of the species in the subgenus Monocalyptus, except Eucalyptus obliqua L'Herit., were associated with negative values on Axis II (Fig. 1). This suggests that Symphyomyrtus and E. obliqua are visited more by birds and butterflies than other Eucalyptus species, which are closer in visitor profiles to the brushlike inflorescences of Melaleuca spp. and Callistemon pallidus (Bonpl.) DC. (Fig. 1). This pattern is much tighter for birds than for butterflies (Table 2). The apparent strong affinity of butterflies for E. globulus Labill. in the ordination plot (Fig. 1) appears to be an artefact of butterflies visiting many other eucalypts also used by birds, and the frequent use of E. globulus by birds, because no butterflies were observed on E. globulus (Table 2).

Visitor profiles of individual plant species also varied between study sites. This was illustrated by L. collinus which was visited almost exclusively by native 
Table 2. Flowering plants, their floral characteristics, and the numbers of anthophile species which they supported

\begin{tabular}{|c|c|c|c|c|c|c|c|c|}
\hline Plants & Colour & Shape & Bee & Wasp & Fly & Beetle & Butterfly & Bird \\
\hline \multicolumn{9}{|l|}{ Aizoaceae } \\
\hline Carpobrotus rossii & pink & actin & 6 & 1 & & & & \\
\hline \multicolumn{9}{|l|}{ Asteraceae } \\
\hline Bedfordia salicina & yellow & actin & 1 & & 1 & 1 & & \\
\hline Brachyglottis brunonis & yellow & actin & & & 1 & & & \\
\hline Cassinia aculeata & pale & actin & 3 & 1 & 2 & 7 & 1 & \\
\hline Erigeron stellatus & pale & actin & & & 1 & & & \\
\hline Helichrysum scorpioides & yellow & actin & 3 & & 2 & 1 & 2 & \\
\hline Olearia phlogopappa & pale & actin & & & 2 & 3 & & \\
\hline Olearia ramulosa & pale & actin & 6 & 1 & 1 & 9 & 3 & \\
\hline Olearia stellulata & pale & actin & 4 & 1 & 1 & 2 & & \\
\hline Olearia viscosa & pale & actin & & & 1 & & & \\
\hline Ozothamnus ferrugineus & pale & actin & 2 & & 2 & 6 & 1 & \\
\hline Ozothamnus obcordatus & pale & actin & 1 & & & 3 & & \\
\hline Ozothamnus thyrsoideus & pale & actin & 1 & & 1 & 1 & 1 & \\
\hline Senecio lautus & yellow & actin & 1 & & 1 & & 1 & \\
\hline Senecio linearifolius & yellow & actin & 2 & & 4 & 2 & 4 & \\
\hline \multicolumn{9}{|l|}{ Campanulaceae } \\
\hline Wahlenbergia gymnoclada & blue & actin & 9 & 1 & 3 & 1 & 3 & \\
\hline \multicolumn{9}{|l|}{ Cunoniaceae } \\
\hline Bauera rubioides & pale & actin & 2 & & 2 & 1 & 1 & \\
\hline \multicolumn{9}{|l|}{ Cyperaceae } \\
\hline Gymnoschoenus sphaerocephalus & pale & brush & & & 2 & 1 & & \\
\hline \multicolumn{9}{|l|}{ Dilleniaceae } \\
\hline Hibbertia fasciculata & yellow & actin & 1 & & & & & \\
\hline Hibbertia procumbens & yellow & actin & & & 1 & & & \\
\hline Hibbertia riparia & yellow & actin & 1 & & & & & \\
\hline \multicolumn{9}{|l|}{ Droseraceae } \\
\hline Drosera arcturi & pale & actin & & & 1 & & & \\
\hline \multicolumn{9}{|l|}{ Epacridaceae } \\
\hline Cyathodes divaricata & pale & tube & & & & & & 2 \\
\hline Cyathodes glauca & pale & tube & 1 & & 7 & 1 & 2 & \\
\hline Cyathodes parvifolia & pale & tube & 5 & 1 & 3 & 3 & & \\
\hline Epacris impressa & pink $^{\mathrm{a}}$ & tube & 4 & & 1 & & 1 & 3 \\
\hline Epacris lanuginosa & pale & tube & 3 & & 3 & & 2 & \\
\hline Epacris serpyllifolia & pale & tube & & & 1 & & & \\
\hline Leucopogon collinus & pale & actin & 8 & 1 & 10 & & 1 & \\
\hline Leucopogon ericoides & pale & actin & 6 & & & 2 & & \\
\hline Leucopogon parviflorus & pale & actin & 1 & & & & & \\
\hline Leucopogon virgatus & pale & actin & 4 & & & 1 & & \\
\hline Lissanthe montana & pale & actin & 1 & & 1 & 1 & & \\
\hline Monotoca glauca & pale & actin & 1 & & 2 & & & \\
\hline Monotoca submutica & pale & actin & 1 & & & & & \\
\hline Pentachondra involucrata & pale & actin & 3 & & 5 & 1 & & \\
\hline Richea pandanifolia & red & actin & & & & & & 1 \\
\hline Richea sprengelioides & pale & actin & & & 1 & & & \\
\hline Sprengelia incarnata & pale & actin & 2 & & & & & \\
\hline \multicolumn{9}{|l|}{ Eucryphiaceae } \\
\hline Eucryphia lucida & pale & actin & 1 & 1 & 10 & 7 & 1 & \\
\hline \multirow{2}{*}{\multicolumn{9}{|c|}{ Fabaceae }} \\
\hline & & & & & & & & \\
\hline Aotus ericoides & yellow & zygo & 13 & & 1 & 1 & 2 & \\
\hline Bossiaea cinerea & yellow & zygo & 4 & & & & & \\
\hline Daviesia latifolia & yellow & zygo & 4 & & 1 & 1 & & \\
\hline Daviesia ulicifolia & yellow & zygo & 2 & & & & & \\
\hline Dillwynia cinerascens & yellow & zygo & 2 & & & & & \\
\hline Dillwynia glaberrima & yellow & zygo & 5 & & & 1 & 1 & \\
\hline Dillwynia sericea & yellow & zygo & 6 & & 1 & 1 & 1 & \\
\hline Gompholobium huegelii & yellow & zygo & 6 & & 1 & & 1 & \\
\hline Oxylobium ellipticum & yellow & zygo & 4 & & 2 & & & \\
\hline Pultenaea daphnoides & yellow & zygo & 4 & & & & & \\
\hline
\end{tabular}


Table 2. continued

\begin{tabular}{|c|c|c|c|c|c|c|c|c|}
\hline Plants & Colour & Shape & Bee & Wasp & Fly & Beetle & Butterfly & Bird \\
\hline Pultenaea gunnii & yellow & zygo & 3 & & & & & \\
\hline Pultenaea juniperina & yellow & zygo & 4 & & & & & \\
\hline Pultenaea pedunculata & yellow & zygo & 2 & & & & & \\
\hline Pultenaea stricta & yellow & zygo & 1 & & & & & \\
\hline \multicolumn{9}{|l|}{ Goodeniaceae } \\
\hline Goodenia lanata & yellow & zygo & & & 1 & & & \\
\hline Goodenia ovata & yellow & zygo & 5 & & 2 & & & \\
\hline $\begin{array}{l}\text { Iridaceae } \\
\text { Patersonia fragilis }\end{array}$ & blue & actin & 1 & & & & & \\
\hline \multicolumn{9}{|l|}{ Lamiaceae } \\
\hline Prostanthera lasianthos & purple & zygo & 4 & 1 & 9 & 4 & 2 & \\
\hline $\begin{array}{l}\text { Westringia rubiaefolia } \\
\text { Lauraceae }\end{array}$ & purple & zygo & 1 & & & & & \\
\hline Cassytha pubescens & pale & actin & 2 & & & & & \\
\hline \multicolumn{9}{|l|}{ Lentibulariaceae } \\
\hline Utricularia dichotoma & purple & zygo & 3 & & 3 & & 1 & \\
\hline \multicolumn{9}{|l|}{ Liliaceae } \\
\hline Arthropodium milleflorum & pale & actin & 2 & & & & & \\
\hline Bulbine bulbosa & yellow & actin & 1 & & 1 & & & \\
\hline Dianella tasmanica & blue & actin & 2 & & & & & \\
\hline \multicolumn{9}{|l|}{ Mimosaceae } \\
\hline Acacia botrycephala & yellow & brush & & & 1 & & & \\
\hline Acacia mearnsii & yellow & brush & 3 & & & 1 & & \\
\hline Acacia sophorae & yellow & brush & 1 & & & & & \\
\hline \multicolumn{9}{|l|}{ Monomiaceae } \\
\hline Atherosperma moschatum & pale & actin & 1 & & & & & \\
\hline \multicolumn{9}{|l|}{ Myrtaceae } \\
\hline Baeckea gunniana & pale & actin & 2 & 1 & 6 & 3 & 1 & \\
\hline Baeckea leptocaulis & pale & actin & 6 & & 2 & 1 & 1 & \\
\hline Baeckea ramosissima & pale & actin & 4 & & & & & \\
\hline Callistemon pallidus & pale & brush & 4 & 1 & & 3 & & \\
\hline Callistemon viridiflorus & pale & brush & 1 & & & & & \\
\hline Eucalyptus amygdalina & pale & brush & 8 & & 3 & 3 & 1 & \\
\hline Eucalyptus coccifera & pale & brush & 12 & 2 & 13 & 6 & & \\
\hline Eucalyptus delegatensis & pale & brush & 1 & 1 & 7 & 5 & & \\
\hline Eucalyptus globulus & pale & brush & 1 & & 1 & 1 & & 3 \\
\hline Eucalyptus johnstonii & pale & brush & 2 & & 10 & 2 & 1 & 3 \\
\hline Eucalyptus obliqua & pale & brush & 2 & 2 & 5 & 1 & 3 & 5 \\
\hline Eucalyptus ovata & pale & brush & 4 & & 5 & 2 & 1 & 2 \\
\hline Eucalyptus pulchella & pale & brush & 3 & & 3 & 2 & & \\
\hline Eucalyptus subcrenulata & pale & brush & & & 4 & & & \\
\hline Eucalyptus urnigera & pale & brush & 6 & & 7 & 2 & & 4 \\
\hline Eucalyptus viminalis & pale & brush & 30 & 8 & 16 & 8 & 2 & 9 \\
\hline Leptospermum glaucescens & pale & actin & 12 & 4 & 16 & 14 & 2 & \\
\hline Leptospermum lanigerum & pale & actin & 17 & 2 & 18 & 11 & 3 & \\
\hline Leptospermum nitidum & pale & actin & 6 & 1 & 10 & 3 & 2 & \\
\hline Leptospermum rupestre & pale & actin & 4 & 2 & 15 & 7 & 1 & \\
\hline Leptospermum scoparium & pale & actin & 28 & 16 & 27 & 13 & 6 & \\
\hline Melaleuca squamea & purple & brush & 4 & & 3 & 1 & & \\
\hline Melaleuca squarrosa & pale & brush & 19 & 2 & 3 & 7 & 1 & \\
\hline \multicolumn{9}{|l|}{ Pittosporaceae } \\
\hline Bursaria spinosa & pale & actin & 11 & 5 & 11 & 10 & 4 & \\
\hline \multicolumn{9}{|l|}{ Proteaceae } \\
\hline Banksia marginata & yellow & brush & 21 & 3 & 6 & 3 & 2 & 5 \\
\hline Hakea lissosperma & pale & actin & 4 & 1 & 5 & & & \\
\hline Lomatia polymorpha & pale & actin & & 1 & 4 & 2 & 1 & \\
\hline Lomatia tinctoria & pale & actin & 5 & & 4 & 5 & 1 & \\
\hline Orites acicularis & pale & actin & & & 1 & & & \\
\hline Orites diversifolia & pale & actin & & 1 & & & & \\
\hline Orites revoluta & pale & actin & 1 & & 4 & & & \\
\hline
\end{tabular}


Table 2. continued

\begin{tabular}{|c|c|c|c|c|c|c|c|c|}
\hline Plants & Colour & Shape & Bee & Wasp & Fly & Beetle & Butterfly & Bird \\
\hline $\begin{array}{l}\text { Persoonia juniperina } \\
\text { Rhamnaceae }\end{array}$ & yellow & actin & 1 & & & & & \\
\hline $\begin{array}{l}\text { Pomaderris elliptica } \\
\text { Rutaceae }\end{array}$ & pale & actin & 2 & & 1 & & & \\
\hline Boronia citriodora & pale & actin & 2 & & 2 & 1 & 1 & \\
\hline Boronia pilosa & pale & actin & 1 & & & & & \\
\hline Correa alba & pale & actin & 3 & & 1 & & 1 & \\
\hline Correa lawrenciana & pale & tube & 1 & & & & & 2 \\
\hline Eriostemon verrucosus & pale & actin & 1 & & & & 1 & \\
\hline Scrophulariaceae & & & & & & & & \\
\hline Euphrasia collina ssp. collina & purple & zygo & 2 & & 1 & & & \\
\hline Euphrasia collina ssp. diemenica & purple & zygo & 5 & & 5 & & & \\
\hline Euphrasia striata & purple & zygo & & & 4 & & 1 & \\
\hline $\begin{array}{l}\text { Veronica formosa } \\
\text { Stylidiaceae }\end{array}$ & purple & zygo & 5 & 1 & 1 & & & \\
\hline $\begin{array}{l}\text { Stylidium graminifolium } \\
\text { Thymeliaceae }\end{array}$ & pink & actin & 11 & & 1 & & 2 & \\
\hline Pimelea humilis & pale & tube & & & 1 & & & \\
\hline Pimelea nivea & pale & tube & & & 2 & & & \\
\hline
\end{tabular}

${ }^{\text {a}}$ Some populations also have white flowers. Colours have been defined as pale if they are white or have pastel tones. Yellow is bright yellow. Blue, purple and pink are bold colours or nectar guides against a pale background. Flowers have been defined as tubular if the corolla tube is longer than the corolla lobes, and brush if the anthers form most of the display. Actin, actinomorphic petaloid including the capitula of Asteraceae; zygo, zygomorphic petaloid.

bees at one coastal heath site, with only one species of fly recorded. However at another area of coastal heath, flies were the most common visitors to this species, with only a few native bees, and one butterfly also observed. In contrast to both of these sites, equal diversities of native bees and flies were recorded on this species in two of the dry sclerophyll forest sites. At one area of coastal heath, Olearia ramulosa (Labill.) Benth. was visited predominantly by bees and beetles, whereas in another coastal heath site no bees were recorded on this species. In an area of dry sclerophyll forest, beetles and butterflies were the major orders supported by $O$. ramulosa, but only one native bee species was recorded. Similarly, Ozothamnus ferrugineus DC. was visited only by beetles in coastal heath, but hosted all four major insect orders in dry sclerophyll forest. Another widespread species, Senecio linearifolius A. Rich. also supported contrasting suites of anthophiles at different sites. In coastal heath the only native visitors that were encountered were flies, whereas at one area of dry sclerophyll forest two species of native bee, one fly and one beetle were recorded from this species. In another dry sclerophyll forest butterflies were the most common taxon visiting this species while the other three major insect orders were also recorded, whereas at higher altitude flies were the most frequent visitors with only one butterfly species observed. Variation in the suites of floral visitors to individual species was also apparent in Banksia marginata Cav. At low altitude, bees were its most frequent visitors, whereas at $1000 \mathrm{~m}$ birds and flies were the most common visitors to this species.

\section{DISCUSSION}

Most of the plant species that were positively associated with the vectors described by flies, beetles, and wasps exhibited floral morphologies approximated by the allophilic syndrome consistent with Faegri and van der Pijl (1979). However, no evidence was obtained of syndromes particular to each of these groups. The lack of floral specialization towards these groups was evident from the similarity of their vectors and the absence of strictly fly-, beetle-, or wasp-pollinated flowers. While this concurs with the absence of a distinct set of floral characters associated with wasps, it is contrary to the concept of specialization towards pollination by flies and beetles (Faegri \& van der Pijl 1979).

An absence of floral specialization towards butterflies was also apparent. In contrast to numerous other studies (reviewed in Armstrong 1979), and our own casual observations at other times, butterflies were not observed on Pimelea spp. during this study, despite them exhibiting the narrow tubular odourless flowers typical of the butterfly syndrome of Faegri and van der Pijl (1979). Flies were the only native insects noted at these flowers (Table 2). (The long-tongued exotic bees from the family Apidae, Apis mellifera L. and Bombus terrestris (L.), were also observed.) Long-tongued bombyliid flies have also been recorded from Pimelea spp. by Armstrong (1979) and, along with longtongued tabanid and nemestrinid flies, from other tubular flowers in the South African fynbos (Johnson 1992). The absence of foraging records of butterflies 
on E. globulus is consistent with a more detailed recent study of its visitors (Hingston \& Potts 1998). The lack of butterfly foraging on this species, in contrast to many of its congeners, may be due to different flowering phenologies. Eucalyptus globulus blooms in spring, while most eucalypts visited by butterflies flower in summer and autumn when most butterflies are volant in Tasmania (McQuillan 1994).

Birds predominantly foraged on tubular and brushlike inflorescences in accordance with the syndrome proposed by Faegri and van der Piil (1979) and with observations in the South African fynbos (Johnson 1992). Foraging on brush-like inflorescences was apparent from the strong affinity of birds with the larger-flowered species of Eucalyptus, especially those in the subgenus Symphyomyrtus. However, these allophilic flowers were also visited by a wide variety of insects (Table 2), indicating a lack of specialization towards birds.

Some specialization towards birds was apparent within a few genera that were otherwise visited by insects. Birds foraged on tubular flowers, while insects were the major visitors to their more allophilic congeners, consistent with the syndromes espoused by Faegri and van der Pijl (1979). Within the genus Correa, the pale yellow tubular flowers of Correa lawrenciana Hook. hosted two species of birds and one species of bee, while the more open white flowers of Correa alba Andr. hosted three species of bees and one each of flies and butterflies (Table 2). Armstrong (1979) also found that C. alba was insect-pollinated whereas all other Correa species, which have tubular flowers, were bird-pollinated. As further evidence, the tubular flowers of Cyathodes divaricata Hook. f., and the purely pink populations of the tubular Epacris impressa Labill. were the only flowers regularly visited by birds but not native insects (Table 2). These epacrid genera both included others with shorter corolla tubes (Curtis 1963), namely C. parvifolia $\mathrm{R}$. Br. and E. lanuginosa Labill., which were visited exclusively by insects (Table 2). In addition, mixed white and pink populations of E. impressa, which exhibit shorter corolla tubes than purely pink populations (Stace \& Fripp 1977b), were visited by both birds and insects. These findings are in accordance with the longer corolla tubes in bird than insect-pollinated species of Erica, in the closely related family Ericaceae, in South Africa (Rebelo \& Siegfried 1985). However, contrary to the syndromes of Faegri and van der Pijl (1979), and to the Erica spp. in South Africa (Rebelo \& Siegfried 1985), the insect-pollinated Cyathodes glauca Labill. has a longer corolla tube than C. divaricata (Curtis 1963).

The observed absence of native insects on purely pink flowered populations of $E$. impress a supports the proposal of Stace and Fripp (1977a) that pink flowers in this species were adapted to bird-pollination, and of Faegri and van der Pijl (1979) who included red colouration as part of their syndrome. However, this situation contrasts with the frequent visits by insects to red and pink flowers in the related genus Erica in South Africa (Rebelo \& Siegfried 1985). Although most flowers visited by birds were pale in colour (Table 2), contrary to the bird-pollination syndrome, Faegri \& van der Pijl (1979) did acknowledge that such situations occur in some regions such as Hawaii.

However, within Epacridaceae, differences in visitor profiles to congeners also occurred in the absence of major differences in floral morphology. Leucopogon collinus (Labill.) R. Br. supported large numbers of bees and flies, whereas all of its congeners were predominantly visited by bees and hosted no flies (Table 2). The observed suite of visitors to $L$. collinus has similarities with that described for this genus by Armstrong (1979), in the form of long-tongued flies from the families Bombyliidae, Calliphoridae, Muscidae, Syrphidae, and Tachinidae. However, only one species of butterfly was recorded from this genus, in contrast to longtubed Leucopogon spp. from the Australian mainland (Armstrong 1979).

The classic bee-pollination syndrome of Faegri and van der Pijl (1979) is plainly evident in Fabaceae. These zygomorphic yellow flowers with nectar guides and concealed rewards were largely visited by bees, as in Spanish shrublands (J. Herrera 1988). This predominance of large bees on Fabaceae supports the claim by Faegri and van der Pijl (1979) that these flowers are adapted to bees which are strong enough to force the wings and keel apart in order to access nectar. However, several common plants displaying this syndrome received very few visitors. These included Indigofera australis Willd. (Fabaceae), Comesperma spp. (Polygalaceae), and the Iridaceae species Diplarrena moraea Labill., Patersonia fragilis (Labill.) Asch. \& Graebner, and Xyris marginata Rendle. The flowers of I. australis are purple, in contrast to the yellow flowers of all other Fabaceae in this study, suggesting that Tasmanian bees may not be attracted to purple zygomorphic flowers. Nevertheless, one specimen of an anthophorid bee in the genus Exoneura Smith was collected from I. australis in a later study (Hingston 1999). That study also revealed that the blue zygomorphic flowers of Comesperma volubile Labill. are visited by similar suites of bees to the majority of yellow-flowered Fabaceae, and that the halictid bees Lasioglossum (Chilalictus) gilesi (Cockerell) and Lasioglossum (Chilalictus) littleri (Cockerell) occasionally visited $D$. moraea (Hingston 1999).

In spite of the tendency for zygomorphic flowers with nectar guides to be visited by bees, this was confounded by differences in the relative abundances of the anthophile groups in various habitats. At high altitude (700-1100 m), where bee diversity was low (Hingston 1998) but flies reached their greatest diversity, non-fabaceous plants exhibiting bee syndromes were 
largely visited by flies. These included E. striata R. Br., E. collina ssp. diemenica Spreng. and Prostanthera lasianthos Labill. Such use of ostensibly melittophilous flowers by flies in cold climates not amenable to bee activity was acknowledged by Faegri and van der Pijl (1979). Dominance of flower visitor guilds by flies at high altitude has also been recorded in Europe (Müller 1880), New Zealand (Primack 1983), Chile (Arroyo et al. 1982), and the USA (Warren et al. 1988; McCall \& Primack 1992). This trend was also apparent in some genera exhibiting allophilic syndromes, such as Lomatia, Olearia, Baeckea, Eucalyptus and Leptospermum with low altitude species attracting many bees while their high altitude congeners with similar floral morphologies were visited predominantly by flies.

Further doubt regarding the applicability of the syndromes espoused by Faegri and van der Pijl (1979) to the Tasmanian flora arises from the considerable number of predominantly bee visited plants which are actinomorphic with exposed rewards. However some of these actinomorphic species (Carpobrotus rossii Schwartes, Stylidium graminifolium Swartz, and Wahlenbergia gymnoclada $\mathrm{N}$. Lothian) do differ from the allophilic syndrome in their bright pink or purple colours. This concurs with the observations by McCall and Primack (1992) of bees contributing to large proportions of the visitors to purple flowers in a Massachusetts woodland meadow, and to pink flowers in South African fynbos scrub. The common association between Tasmanian bees and flowers with exposed rewards can be attributed to the predominance of short-tongued bees in Tasmania, while the melittophilous syndrome of Faegri and van der Pijl (1979) is specific to the long-tongued Apidae. The use of allophilic flowers by short-tongued bees is most apparent in the Colletidae, which forage heavily on Myrtaceae (Armstrong 1979; Hingston 1999). In contrast, many bees in the other short-tongued family in Tasmania, Halictidae, visit Fabaceae (Hingston 1999). As the Colletidae are more diverse in Australia than any other continent (Armstrong 1979), the use of allophilic flowers by bees is likely to be more frequent than elsewhere.

The usefulness of pollination syndromes in Tasmania is limited because most plant species host a taxonomically diverse array of visitors. This result is consistent with previous community-level studies in Mediterranean climates of the northern hemisphere (J. Herrera 1988; C. Herrera 1996; Bosch et al. 1997). This absence of specialization by plants to particular pollinators has been attributed to the evolutionary risk associated with dependence on another species (Boucher et al. 1982; Johnson 1992; Waser et al. 1996), and to the unpredictability of pollinator populations in both time and space (C. Herrera 1988, 1996; Pettersson 1991; Johnson 1992; Waser et al. 1996).
Fluctuations in abundances of anthophile taxa between years have been noted in other studies (Schemske \& Horvitz 1988; Heard et al. 1990; Eckhart 1992), and spatial variation was apparent in this study as well as others (C. Herrera 1988; Eckhart 1992). Hence, generalist pollination systems are advantageous in ensuring pollination in the event of environmental change (J. Herrera 1988), and assist plants to expand their ranges into new habitats (Ghazoul 1997). It is notable that plants frequently adopt generalist pollination systems in the relatively unpredictable Mediterranean-type climates (J. Herrera 1988; C. Herrera 1996; Bosch et al. 1997), whereas specialized systems appear to be quite common in the tropics (Armstrong \& Irvine 1989; Gautier-Hion \& Maisels 1994; Ghazoul 1997; Momose et al. 1998). Recurrent fires, of variable frequency and intensity, which cause unpredictable changes in plant assemblages in Mediterranean-type climates have also been suggested as contributing to selection for generalized plantpollinator relationships (Johnson 1992).

This study has found that the diurnal pollination syndromes espoused by Faegri and van der Pijl (1979) are unreliable predictors of floral visitors in Tasmania. While the bee- and bird-pollinated syndromes are sometimes valid, the butterfly, fly and beetle syndromes are largely inapplicable to the Tasmanian flora. However, the results are based on presence/absence of particular visitors and the inclusion of uncommon visitors may make plants appear less specialized than would be the case if frequencies of visits were taken into account. Further research is required to test the applicability to Tasmania of the pollination syndromes associated with nocturnal animals: mothpollination (phalaenophily) and mammal-pollination (therophily).

\section{ACKNOWLEDGEMENTS}

We thank the Tasmanian Department of Environment and Land Management via M. Driessen, World Heritage Area Zoologist, for financial assistance. The project was also funded by a University of Tasmania scholarship to $\mathrm{ABH}$. Much gratitude also goes to $\mathrm{Dr}$ Ken Walker of the Museum of Victoria for access to his bee collection, and for providing many species identifications.

\section{REFERENCES}

Armstrong J. A. (1979) Biotic pollination mechanisms in the Australian flora - a review. N.Z. F. Bot. 17, 467-508.

Armstrong J. E. \& Irvine A. K. (1989) Floral biology of Myristica insipida (Myristicaceae), a distinctive beetle pollination syndrome. Am. F. Bot. 76, 86-94. 
Arroyo M. T. K., Primack R. \& Armesto J. (1982) Community studies in pollination ecology in the high temperate Andes of central Chile. I. Pollination mechanisms and altitudinal variation. Am. F. Bot. 69, 82-97.

Belbin L. (1993) Users Guide. PATN Pattern Analysis Package. CSIRO Division of Wildlife \& Ecology, Canberra.

Borror D. J., Long D. M. \& Triplehorn C. A. (1981) An Introduction to the Study of Insects. Saunders College Publishing, Philadelphia.

Bosch J., Retana J. \& Cerda X. (1997) Flowering phenology, floral traits and pollinator composition in a herbaceous Mediterranean plant community. Oecologia 109, 583-91.

Boucher D. H., James S. \& Keeler K. H. (1982) The ecology of mutualism. Ann. Rev. Ecol. Syst. 13, 315-47.

Buchanan A. M. (1995) A Census of the Vascular Plants of Tasmania. The Tasmanian Herbarium, Hobart.

Cruden R. W. \& Hermann-Parker S. M. (1979) Butterfly pollination of Caesalpinia pulcherrima, with observations on a psychophilous syndrome. F. Ecol. 67, 155-68.

Curtis W. M. (1963) The Student's Flora of Tasmania. Part 2. University of Tasmania, Hobart.

Curtis W. M. (1967) The Student's Flora of Tasmania. Part 3. University of Tasmania, Hobart.

Curtis W. M. \& Morris D. I. (1975) The Student's Flora of Tasmania. Part 1. University of Tasmania, Hobart.

Curtis W. M. \& Morris D. I. (1994) The Student's Flora of Tasmania. Part 4B. St David's Park Publishing, Hobart.

Eckhart V. M. (1992) Spatio-temporal variation in abundance and variation in foraging behaviour of the pollinators of gynodioecious Phacelia linearis (Hydrophyllaceae). Oikos 64, 573-86.

Faegri K. \& van der Pijl L. (1979) The Principles of Pollination Ecology. Pergamon Press, Oxford.

Gautier-Hion A. \& Maisels F. (1994) Mutualism between a leguminous tree and large African monkeys as pollinators. Behav. Ecol. Sociobiol. 34, 203-10.

Ghazoul J. (1997) The pollination and breeding system of Dipterocarpus obtusifolius (Dipterocarpaceae) in dry deciduous forests of Thailand. F. Nat. Hist. 31, 901-16.

Heard T. A., Vithanage V. \& Chacko E. K. (1990) Pollination biology of cashew in the Northern Territory of Australia. Aust. F. Agric. Res. 41, 1101-14.

Herrera C. M. (1988) Variation in mutualisms: the spatiotemporal mosaic of a pollinator assemblage. Biol. F. Linn. Soc. 35, 95-125.

Herrera C. M. (1996) Floral traits and adaptation to insect pollinators: a devil's advocate approach. In: Floral Biology (eds D. G. Lloyd \& S. C. H. Barrett) pp. 65-87. Chapman \& Hall, New York.

Herrera J. (1988) Pollination relationships in southeastern Spanish Mediterranean shrublands. f. Ecol. 76, 274-87.

Hingston A. B. (1998) Temporal and spatial variation in abundances of native bee species on an altitudinal gradient in southern Tasmania. Aust. F. Zool. 46, 497-507.

Hingston A. B. (1999) Affinities between southern Tasmanian plants in native bee visitor profiles. Aust. F. Zool. 47, 361-84.
Hingston A. B. \& Potts B. M. (1998) Floral visitors of Eucalyptus globulus ssp. globulus in eastern Tasmania. Tasforests 10, 125-37.

Johnson S. D. (1992) Plant-animal relationships. In: The Ecology of Fynbos: Nutrients, Fire and Diversity (ed. R. M. Cowling) pp. 175-205. Oxford University Press, Cape Town.

Kirk W. D. J. (1988) Assessing the effects of flower thrips. Acta Phytopathol. Entomol. Hungary 23, 295-300.

McCall C. \& Primack R. B. (1992) Influence of flower characteristics, weather, time of day, and season on insect visitation rates in three plant communities. Am. F. Bot. 79, 434-42.

McQuillan P. B. (1994) Butterflies of Tasmania. Tasmanian Field Naturalists Club, Hobart, Tasmania.

Michener C. D. (1965) A classification of the bees of the Australian and South Pacific regions. Bull. Am. Mus. Nat. Hist. 130, 1-362.

Momose K., Yumoto T., Nagamitsu T et al. (1998) Pollination biology in a lowland dipterocarp forest in Sarawak, Malaysia. I. Characteristics of the plant-pollinator community in a lowland dipterocarp forest. Am. F. Bot. 85, 1477-501.

Müller H. (1880) The fertilisers of alpine flowers. Nature 21, 275.

Pettersson M. W. (1991) Pollination by a guild of fluctuating moth populations: option for unspecialization in Silene vulgaris. F. Ecol. 79, 591-604.

Primack R. B. (1983) Insect pollination in the New Zealand mountain flora. N.Z. F. Bot. 21, 317-33.

Rebelo A. G. \& Siegfried W. R. (1985) Colour and size of flowers in relation to pollination of Erica species. Oecologia 65, 584-90.

Rourke J. \& Wiens D. (1977) Convergent floral evolution in South African and Australian Proteaceae and its possible bearing on pollination by nonflying mammals. Ann. Mo. Bot. Garden 64, 1-17.

Rozzi R., Arroyo M. K. \& Armesto J. J. (1997) Ecological factors affecting gene flow between populations of Anarthrophyllum cumingii (Papilionaceae) growing on equatorial- and polarfacing slopes in the Andes of Central Chile. Plant Ecol. 132, 171-9.

Schemske D. W. \& Horvitz C. G. (1988) Plant-animal interactions and fruit production in a neotropical herb: a path analysis. Ecology 69, 1128-37.

Stace H. M. \& Fripp Y. J. (1977a) Raciation in Epacris impressa. I Corolla colour and corolla length. Aust. F. Bot. 25, 299-314.

Stace H. M. \& Fripp Y. J. (1977b) Raciation in Epacris impressa III Polymorphic populations. Aust. F. Bot. 25, 325-36.

Walker K. L. (1995) Revision of the Australian native bee subgenus Lasioglossum (Chilalictus) (Hymenoptera: Halictidae). Mem. Mus. Vic. 55, 1-423.

Warren S. D., Harper K. T. \& Booth G. M. (1988) Elevational distribution of insect pollinators. Am. Midl. Nat. 120, 325-30.

Waser N. M., Chittka L., Price M. V., Williams N. M. \& Ollerton J. (1996) Generalization in pollination systems, and why it matters. Ecology 77, 1043-60.

Wyatt R. (1981) Ant-pollination of the granite outcrop endemic Diamorpha smallii (Crassulaceae). Amer. f. Bot. 68, 1212-7. 